\section{Assessment of pesticides}

The Use and Significance of Pesticides in the Environment. By F. McEwen and G. Stephenson. Pp.538. (Wiley: New York and Chichester, UK, 1979.) £16.75.

THIS book is based on lectures for a course that the authors have run for several years at the University of Guelph, and it is intended to give senior undergraduates a broad assessment of pesticides. The authors have achieved a high degree of detached assessment, based on the premise that although pesticides are virtually essential they also can and do present serious problems. On occasion this detachment almost becomes a fault, as both sides of some issues are presented in such a way that the authors' opinion is not immediately obvious. Students are unlikely to have the background of knowledge and experience to decide for themselves what reasonable assumptions can be drawn from a series of disjointed facts unless there is a fairly clear critical assessment of the issues involved.

The introductory chapter outlines the development of modern synthetic insecticides during and since the Second World War, the consequent problems of resistance to insecticides, the contamination of the environment by persistent insecticides, the effects on wildlife, possible hazards to human beings, and the possible role of integrated control. Several brief chapters then describe the types of issue raised by the use of pesticides, their use in public health, agriculture, forestry and 'rights-of-way', and for domestic purposes. No mention is made of industrial uses, particularly the treatment of wool and timber, which is causing much discussion in some parts of the world. A brief elementary but good chapter describes current testing procedures for the development of new pesticides: it should perhaps have referred to the current pressure for increasingly extensive and expensive toxicity tests, with the possible repercussions on the ability and willingness of chemical companies to develop new pesticides.

The major part of the book falls into two sections. The first gives brief accounts of the chemical, physical and biological properties of many individual pesticides, and the second describes the occurrence, movement and effects of pesticides in various parts of the environment - soil, water, air, food, birds, mammals and the biosphere.

This is a book that requires intelligent skimming, which would be helped if each chapter started with a list of its subdivisions. There is much material that may be useful for reference, but surely not all to be considered in detail. Examples are taken principally from North America, but this does not altogether detract from the book's value for a British or European readership. For instance, the accounts of spraying against spruce budworm in the forests of New Brunswick, the consequent effects on salmon in the Miramichi River, and the effects of parathion on agricultural workers in California are all of general interest.

I would fault the book on details. The section that describes individual pesticides, 'despite its extensive coverage, is not too well integrated with the section on pesticides in the environment: residues of dichlobenil and BHC are mentioned, but the student will find no account of their chemistry and properties. The decline of peregrine falcons (Falco peregrinus) in Great Britain is attributed to eggshell thinning, instead of the acute toxicity of dieldrin poisoning. The authors do state that pesticides in water can be associated with particulate matter, but quote $\mathrm{LC}_{90}$ values for DDT of up to 2,400 p.p. $10^{9}$, whereas the best estimates for the maximum solubility of DDT in water are a little over 1 p.p. $10^{9}$. Similarly no comment is made on this aspect of Wurster's work on the effects of DDT on phytoplankton at concentrations of 10-100 p.p. $10^{9}$.

On occasion too one would like the authors' opinions. The Delaney amendment in the USA, which prohibits the use on food of any pesticide that has been shown to be carcinogenic, is mentioned without any comment, although eighty pages later two and a half pages are devoted to testing for carcinogens. Again we are told, without comment, that "environmentalists" have criticised severely the use of dieldrin against fire ants. This is a programme of which those outside of North America may have heard but may lack detailed knowledge.

There is remarkably little about resistance - it is not listed in the index at all - nor about the wider ecological implications of pesticide use. To conclude, this is a book worth dipping into, although it will not suffice on its own for a broad assessment of pesticides.

F. Moriarty

F. Moriarty is a Principal Scientific Officer at Monks Wood Experimental Station, Institute of Terrestrial Ecology, Abbots Ripton, UK.

\section{Crystal structure determinations}

Inorganic Molecular Dissymmetry. By Y. Saito. Pp. 167. (Springer: Berlin, Heidelberg and New York, 1979.) \$42.90; DM78.

PROFESSOR Yoshihiko Saito pioneered in 1955 the determination of the absolute stereochemical configuration of chiral transition metal coordination compounds by the anomalous $X$-ray scattering method, following the application of the technique by Bijvoet in 1951 to (+)-tartaric acid. The latter study showed that the arbitrary stereochemical convention of Emil Fischer, proposed in 1891 , is in fact correct, while the former demonstrated that Werner's prototype dissymmetric complex, (+)-tris(ethylenediamine)cobalt(III), (+)-[Co(en $\left.)_{3}\right]^{3+}$, has the morphology of a left-handed threebladed propeller, later termed the $\boldsymbol{A}$-configuration.

More recently Professor Saito has measured the electron-density distribution around the metal ion in transition metal complexes from the difference synthesis maps obtained through the use of automated X-ray diffractometers. In the case of $\left[\mathrm{Co}(\mathrm{en})_{3}\right]^{3+}$, for example, it is found that the cobalt(III) ion is almost uncharged, in agreement with Pauling's electroneutrality principle, and that the 3d-electron density distribution is aspheric, with lobes located at the corners of a cube centred on the metal ion, while the electron density of the nitrogen non-bonding electron-pairs forming the coordinate bonds are somewhat displaced from the line joining the nitrogen atom to the metal ion.

His book, Inorganic Molecular Dissymmetry, provides a concise account of the principles underlying these studies and of their application in selected crystalstructure determinations, together with the theoretical basis and the practical articulation of conformational analysis applied to metal chelates, and of the investigation of d-electron optical activity in chiral transition metal complexes. The introduction to the book covers the types of isomerism encountered in metal complexes, and used by Werner in his structure theory, symmetry notation, and the specification of absolute configuration. The illustrations are clear and instructive, as are the mathematical appendices.

The book is directed towards a general and synthetic understanding of chiral molecules, and their unique property of optical activity, in the field of transition metal chemistry. The level of treatment is suited to graduate or advanced undergraduate teaching. For these roles, and for library reference, the book is strongly recommended.

S.F. Mason

S.F. Mason is Professur of Chemistry at King's College, University of London, UK. 